\title{
À propos de l'exposition « Nouvelle-Irlande » au musée du quai Branly et de son catalogue
}

\section{Gilles Bounoure}

\section{(2) OpenEdition \\ 1 Journals}

Édition électronique

URL : http://journals.openedition.org/jso/1044

DOI : $10.4000 /$ jso. 1044

ISSN : $1760-7256$

Éditeur

Société des océanistes

\section{Édition imprimée}

Date de publication : 1 décembre 2007

Pagination : 321-327

ISBN : 978-2-85430-010-9

ISSN : 0300-953x

Référence électronique

Gilles Bounoure, «À propos de l'exposition « Nouvelle-Irlande » au musée du quai Branly et de son catalogue », Journal de la Société des Océanistes [En ligne], 125 | Année 2007-2, mis en ligne le 02 juillet 2008, consulté le 19 avril 2019. URL : http://journals.openedition.org/jso/1044 ; DOI : 10.4000/ jso. 1044

\section{(c) Tous droits réservés}




\section{MISCELLANÉES}

\section{À propos de l'exposition « Nouvelle-Irlande » au musée du quai Branly et de son catalogue}

par

\section{Gilles BOUNOURE*}

Quoiqu'on puisse soutenir, avec Victor Hugo, qu' « une tour, c'est le contraire d'un puits », et un lac ou un fleuve, "le contraire d'une île» (Lucrèce Borgia), il y a au moins deux similitudes directes entre le bassin du fleuve Sépik aux centaines d'affluents et l'archipel néo-irlandais aux cent-cinquante îles : ce furent les deux foyers les plus fameux et productifs qu'aient comptés les arts mélanésiens, et la diversité des styles ayant trouvé à s'y épanouir, de mieux en mieux reconnue au fil des études de plus en plus serrées qui leur sont consacrées, est là pour dissuader de plus en plus fermement les spécialistes occidentaux, y compris les meilleurs, de porter un regard complet sur l'une ou l'autre de ces aires artistiques, dont la formation, pourtant, ne doit pas tout à la géographie physique. On se souvient que pour les arts du Sépik, Douglas Newton avait entrepris de relever le défi d'une description exhaustive, que la mort l'a empêché de mener jusqu'à son terme. Pour ceux de la NouvelleIrlande, Michael Gunn travaille depuis plus de trois décennies à un projet d'une ampleur et d'une ambition similaires, et la manifestation du quai Branly, ainsi que son catalogue, derniers fruits de son énergie, représentent un pas important en direction de cette "synthèse » vivement attendue de ceux qui suivent ses travaux ou s'intéressent aux arts océaniens.

Destiné à accompagner l'exposition itinérante qui s'est arrêtée au musée du quai Branly du 3 avril au 8 juillet 2007 (partie du Saint Louis Art Museum, 15 octobre 2006-7 janvier 2007, et finalement présentée à l'Ethnologisches Museum de
Berlin, 10 août-11 novembre 2007), nourrie des collections des musées qui l'accueillaient et d'une vingtaine d'autres institutions du monde entier, et très favorablement accueillie de la presse et du public français pour d'éclatantes raisons sur lesquelles on reviendra, l'ouvrage qu'il a dirigé (Gunn et Peltier, 2007) est certainement celui qui à ce jour rend le mieux justice aux arts néo-irlandais. Le catalogue conçu pour le musée de Minneapolis par Louise Lincoln (1987) ne présentait qu'une cinquantaine d'objets et un nombre équivalent de documents photographiques. Arts rituels d'Océanie. Nouvelle-Irlande dans les collections du musée Barbier-Mueller, rédigé par Michael Gunn (1997), reproduisait quarante-trois objets et quatre-vingt une figures illustrées. On ne sait ce qu'aurait été l'inventaire des collections du musée de Berlin entrepris sur les directives de Gerd Koch si son rédacteur Klaus Helfrich était allé au bout de sa tâche, mais le seul volume paru sur les trois annoncés (Malanggan I, 1973) n'offre que douze planches en couleurs et cent-cinquante-quatre photos noir et blanc mal adaptées à des objets si vivement colorés. Avec cent-trente et une planches et cinquante-trois autres illustrations le plus souvent en couleurs, le catalogue conçu par Michael Gunn et Philippe Peltier s'impose par conséquent comme le plus riche répertoire iconographique jamais imprimé sur les arts de cet archipel.

S'en tiendrait-il à ces images, bien choisies pour souligner cet état de fait, aucun lecteur ne saurait ignorer désormais la diversité de ces arts, que rappelle encore la disposition en six

* flongue@wanadoo.fr 
chapitres principaux, croisant les approches géographiques et thématiques, des cinquante-huit textes (sans compter les notices) dont huit au moins apportent des éclairages différents sur un même sujet. Cela ne va pas sans discordances ou contradictions, certainement délibérées, et la rigueur des maîtres d'œuvre de ce catalogue est aussi de n'avoir pas tenté de les occulter $^{1}$. Par exemple, la notice de la planche 70 , p. 198, concernant une pirogue malangan (conservée au Linden-Museum de Stuttgart) décrit ses extrémités «en forme de poisson ou de requin ", expression également utilisée pour la légende de la frise figurée planche 77 p. 208, tout comme la figure 30, p. 124 est censée correspondre à des "requins de pierre", alors que Michael Gunn, principal contributeur de l'ouvrage, assure que "l'absence de représentation de requins dans le malagan" tient au «caractère sacré de cet animal interdisant de le figurer dans ce système rituel » (p. 200). On citera seulement, parmi maintes opinions différentes, l'observation de Norbert Jacques (1922:105), relevant sur les sculptures malangan qu'il a vues à Tabar, en 1913, que « zwischen den Beinen der Männer wuchsen gewichtige stilisierte Haifische oder Eidechsen gebogen auf » ou le livre récent de Susanne Küchler (2002: 117), qui recense Bewue, le requin, parmi vingtsix autres motifs de l'iconographie malangan.

L'un des grands apports de ce catalogue, et probablement même une révélation pour le grand public et la plupart des amateurs d'art mélanésien habitués par nombre d'expositions à ne connaître de la Nouvelle-Irlande que l'« art malangan », est l'attention remarquable qu'il réserve aux expressions artistiques de la moitié sud de l'archipel. Même pour les connaisseurs, comme le souligne justement Philippe Peltier à propos de Lihir (p. 156), elles présentent de nombreux « points aveugles » ou zones grises, ouvertes à beaucoup de confusions. Une " somme " récente n'hésitait pas, sur la foi de ressemblances superficielles, à attribuer une sculpture de pierre à la société Iniet des Tolai de Nouvelle-Bretagne, malgré les évidences stylistiques et les indications mêmes de son collecteur, le très rigoureux Nicolaï Mikloukho-Maklaï, assurant l'avoir recueillie en Nouvelle-Irlande (Meyer, 1995: 364-365, $\mathrm{n}^{\mathrm{o}} 398$, repris dans Nicolas et al., 2000 : $\left.328-319, \mathrm{n}^{\circ} 65\right)$. D'autres objets sont de prove- nance encore moins assurée: un masque aujourd'hui au musée du quai Branly (Inv. 72.1983.2.1, pp. 157-159), importé en Europe par les missionnaires d'Hiltrup, vendu par un marchand de Hambourg à un autre d'Amsterdam, arrivé sans autres précisions entre les mains de divers marchands parisiens, ne reçut son attribution aux îles Lihir que des gens de musée qui firent son acquisition, à côté d'autres avis autorisés lui assignant plutôt comme origine la région de Muliama, sur l'île principale. C'est sans doute aussi pourquoi Philippe Peltier ne fait pas mention d'objets d'origine encore plus obscure, des masques ou "masques-heaumes" monoxyles, censément collectés en 1970 à Lihir (vente Loudmer, Hôtel Drouot, Paris, $1^{\text {er }}$ juillet 1994 : lot 475, et vente Sotheby's, Galerie Charpentier, Paris, 3 décembre 2004 : lot 22). Restés en mains privées, ils pourraient constituer les seuls vestiges existants de la sculpture de ce groupe d'îles, pour autant qu'ils en soient réellement originaires.

La prudence n'est pas moins de mise à propos d'une série de figurations anthropomorphes sculptées dans le calcaire récemment apparues sur le marché international de l'art, et dont le catalogue reproduit un exemplaire acquis par le Linden-Museum (pp. 126-7, pl. 36). Les premières à être publiées, censément achetées en septembre 2001 dans une brocante de Melbourne auprès de la veuve d'un fonctionnaire affecté en Nouvelle-Irlande avant la Deuxième Guerre mondiale (The Age, Melbourne, 2 avril 2002), amenèrent le Museum Security Network et d'autres organisations analogues à signaler ce « cultural property incident » à leurs correspondants. De fait, sur certaines de ces pièces passées sur le marché parisien, des mousses ou des lichens, et une odeur qu'on aurait pu dire troglodytique, s'accordaient mal avec des décennies de séjour dans une grande ville australienne. L'apparition sur le marché d'autres pièces similaires n'a pas mieux accrédité le "récit d'origine » des cinq sculptures « exhumées » lors de la brocante faramineuse de Melbourne, mais elle fait attendre de nouvelles découvertes de même importance, dans des conditions qu'on souhaiterait mieux justifiables. Selon le même quotidien de Melbourne (The Age, 9 octobre 2003), la Nouvelle-Irlande centrale, d'où proviennent certainement ces objets, venait de voir passer, entre

1. Un souci d'unité fait néanmoins adopter à l'ensemble du catalogue la graphie anglo-saxonne «malagan » qui n'est pas mieux justifiée que les graphies germanique ou française plus anciennes, "malanggan, malangan », utilisées du reste par la plupart des contributeurs dans leurs précédentes publications (Michael Gunn le premier). On s'en tient ici à la dernière graphie, consacrée par l'usage de ce pays-ci. On regrette que le catalogue, pourtant disert sur les implications de ce terme, n'en explicite pas les diverses significations comme l'a fait Dieter Heintze (2003 : 155-194, spécialement 160 sq. : « Malagan : Was ist das ?»). Cet important article manque à la bibliographie du catalogue. 
autres «archéologues amateurs", les troupes gouvernementales s'entraînant en vue d'un débarquement contre les rebelles de Bougainville, ainsi que de nombreux " chercheurs d'or" en quête des lingots qu'y aurait dissimulés l'armée japonaise dans une grotte qu'elle aurait ensuite minée.

Les traits stylistiques de ces pierres sculptées en bas et moyen relief rappellent largement certains relevés d'Emil Stephan dans la région de King, au sud-ouest de l'île principale (voir Südseekunst et surtout Neu-Mecklenburg, 1907 : 116 fig. 122, sur les peintures appliquées aux cadavres) mais aussi ceux de la "trouvaille de Loloba », lot de pierres sculptées collecté en 1908 par le médecin colonial Born sur la côte est de la Nouvelle-Irlande centrale, envoyé aussitôt au musée de Leipzig, publié en 1911 par Gustav Antze et qu'on a longtemps cru broyé sous les ruines du musée bombardé en 1943, avant qu'il soit retrouvé par les soins de Marion MelkKoch, responsable des collections océaniennes de ce musée. Il n'est pas sûr que " toutes les figures en pierre aient été faites dans un calcaire tendre que l'on trouve dans le lit des rivières de la zone montagneuse proche de la région de Punam » (Gunn, p. 123), dernier lieu connu de production de sculptures monolithes en Nouvelle-Irlande. Les sources d'approvisionnement furent sans doute diverses, pierres roulées des torrents comme en observa le missionnaire Danks le 7 avril 1888 sur la côte proche de Kudukudu (1933 : 250, "I then took a piece of chalkstone, out of which the images are made") ou encore gisements exploités en carrière, au rapport du géologue strasbourgeois Karl Sapper, cité par Augustin Krämer (in Elisabeth Krämer-Bannow, 1916 : 272, "an den Kreidelagern [...], aus denen die bekannten Totenfiguren geschnitten werden"). Comment leurs différences d'aspect, de matériau sans doute aussi, pourraient être mises en relations avec leurs différences probables d'époque, de provenance et peutêtre également d'usage rituel, c'est l'un des défis que l'ensemble des sculptures de pierre de
Nouvelle-Irlande pose encore à la recherche moderne.

Pour les autres « raisons historiques" plus notables susceptibles d'expliquer "partiellement » ce genre de « point aveugle » $(\mathrm{Ph}$. Peltier, l.c.), le catalogue se limite malheureusement à un exposé rapide sur la présence coloniale allemande (1885-1914) évoquant pudiquement, pour la partie nord de l'île principale « les bouleversements rapides dus à la nouvelle route » côtière ordonnée par Boluminski (Markus Schindlbeck, p. 33, Ph. Peltier ayant raison de mentionner p. 20, au détour d'un autre exposé, les « déplacements de population par le pouvoir colonial allemand », à l'intérieur de l'archipel comme en direction d'autres colonies, et qui affectèrent évidemment aussi les arts). Sur l'administration australienne de l'archipel (1914-1942), la douloureuse occupation japonaise (1942-1945) et les développements historiques plus récents, le soin est laissé aux lecteurs de s'informer par eux-mêmes, par exemple d'après l'exposé géographiquement limité mais très suggestif de Robert J. Foster (1995 : 25-66). Quant aux bouleversements dus à la guerre du Pacifique, comme l'occupation de Nissan par les troupes américaines et néo-zélandaises, le 14 février 1944, qui en évacuèrent toute la population pour y installer une vaste base aéronavale dont le plus gros du matériel fut jeté à l'issue du conflit dans le lagon transformé en immense décharge, ils peuvent expliquer au moins autant que les "variations de la mode» et les "préférences individuelles » l'« évolution stylistique » (Antje Denner, p. 147, résumant Nachman), ou plutôt la rupture constatée entre les masques des collections occidentales, collectés avant 1914, et ceux dont on gardait le souvenir à Nissan au début des années 1970.

Il n'y a évidemment pas lieu d'exiger des développements encyclopédiques d'un catalogue d'exposition s'adressant au grand public comme aux spécialistes ${ }^{2}$ et principalement destiné à décrire et à éclairer un choix d'objets circonstanciel, dépendant à la fois de l'intérêt iconogra-

2. Sans malheureusement éviter, sur d'infimes détails, des discussions "savantes » indifférentes aux lecteurs de toute compétence ou qualification. M. Gunn (p. 172, note 1) reprend ainsi Christian Kaufmann, pour avoir désigné les sculptures uli du nom erroné de nalik dans ses notices de L'Art océanien (Newton et al., 1993 : 567 et fig. 534-536, p. 463). Il aurait pu ajouter que la légende de la fig. 537, p. 464 du même ouvrage attribue également à la « Nouvelle-Bretagne » la barque malangan du Linden-Museum déjà mentionnée. Erreurs vénielles et peut-être difficilement évitables dans une somme embrassant un si vaste éventail de cultures et d'objets, mais un ouvrage aussi « pointu » que ce catalogue-ci n'y échappe pas. M. Gunn écrit par exemple (p. 160 note 2) : «Quand Richard Parkinson publia sa carte de la Nouvelle-Irlande en 1907, l'île la plus méridionale des Tabar (Big Tabar) était connue des Occidentaux sous le nom de Gardener Insel (île des Jardiniers) » etc. (sic, et non « du Jardinier », ou « Gärtner », ou « Island », ce qui eût été plus cohérent au moins linguistiquement). Il suffit de se référer au livre de Richard Parkinson (1907: 250 et carte entre les pp. 248 et 249) pour constater que cet auteur identifie très clairement Tabar parmi les Gardnerinseln. Comme celui de Tatau, le nom de Tabar est cité par maintes autres sources datant des environs de 1907, notamment la carte accompagnant l'article de Fritz Krause (1907 : 47) mentionné par la bibliographie du catalogue. L'origine de ce nom et sa déformation depuis Tasman jusqu'au XIX ${ }^{\mathrm{e}}$ siècle étaient déjà convenablement expliquées dans l'ouvrage classique (et toujours utile !) de Carl E. Meinicke (1875: I, 141 et notes 367-8) 
phique ou historique des pièces à exposer et des " problèmes d'intendance ", impubliables mais décisifs, entourant toujours le prêt, le transport et l'exposition de vestiges artistiques particulièrement fragiles puisque conçus souvent pour la durée d'une seule cérémonie. Ce sont sans doute ces dernières raisons qui ont empêché le panorama des arts néo-irlandais proposé par les auteurs, déjà très riche, d'être plus complet et équilibré. Quoique les textes le mentionnent au passage, le catalogue (hormis une photographie ancienne, p. 39, un masque, pl. 8, une coiffe, pl. 9) laisse dans l'ombre un centre de style important, non seulement géographiquement, l'île de Lavongai ou Nouveau-Hanovre couvrant un huitième de la superficie totale de l'île principale, mais aussi par ses productions, et les questions qu'elles suggèrent en termes d'influence ou de sources. Par exemple, si ses " masques blancs" ont pu être confondus, en Occident, avec ceux du centre de l'île (excellemment figurés quant à eux pl. 3 et 4), cette erreur fréquente n'est peut-être pas sans fondement, tant il y eut d'échanges de traits stylistiques (et sans doute de rituels) dans l'archipel. Les mêmes raisons expliquent probablement aussi que ne soient ni représentés ni décrits, parmi d'autres types les plus connus d'objets dits « malangan », ceux qui figurent des joueurs de flûte de Pan (voir par exemple Lincoln, Assemblage... o.. c., $\mathrm{n}^{\circ}$ 28, pp. 124-126) identifiés « classiquement», depuis A. Krämer (1925: 49-50, et. pl. 49-51), comme des magiciens faiseurs de pluie ou de soleil. On en dirait autant des «boucliers de danse» (Tanzschilde) auxquels Dieter Heintze avait consacré tout un chapitre de sa remarquable thèse (1969: 90-128) si le catalogue et l'exposition n'incluaient un tel objet (Field Museum, Chicago, 98641, pl .97) sans l'identifier autrement que comme « sculpture malagan asymétrique ", malgré la poignée nettement visible au revers. Mais ce catalogue ne visait pas à dresser une typologie exhaustive, et ses oublis certainement inévitables sont largement contrebalancés par l'importance et le nombre de ses apports, de ses rappels ou de ses mises au point.

En montrant certainement pour la première fois de façon aussi abondante et à un public aussi vaste un génie artistique jadis multiforme, foisonnant et de nature à faire honte à un Occident colonisateur, unificateur et stérilisant, cette exposition et ce catalogue signalent en effet quoique avec une netteté inégale - deux des difficultés principales posées par la plupart des arts dits « primitifs », mais plus spécialement par les arts néo-irlandais du fait de leur diversité énigmatique, si déroutante pour les Occidentaux. En premier lieu, il est extrêmement aventureux, à partir ou auprès de sociétés très jalouses de leurs spécificités (et les individus qui les composaient n'étaient pas moins sourcilleux sur leurs propres particularités, comme l'ont heureusement mis en évidence des recherches récentes), d'extrapoler sur le compte de sociétés voisines animées des mêmes dispositions. Tout comme la plus complète description ethnographique jamais publiée sur la Nouvelle-Irlande, celle d'Hortense Powdermaker (Life in Lesu, 1933), ne vaut que pour un petit district à un bref moment de son histoire, l'ethnologue finissant aussi par reconnaître avec une remarquable lucidité sa «fausse position» parmi les insulaires («phoniness », écrit-elle dans Stranger and Friend, 1966 : 119), de même les recherches récentes nourrissant plus ou moins directement ce catalogue (M. Gunn pour les îles Tabar, S. Küchler pour les environs de Lamusmus, Sean Kingston pour la région de Siar, Brigitte Derlon pour le plateau Lelet, mais aussi celles de Brenda Johnson Clay en pays mandak et de Roy Wagner en pays barok, pour ne citer que les plus en vue) restent apparemment incapables de fournir des vues généralisables à l'ensemble des arts ou même des modes de vie et de pensée néo-irlandais précédant la colonisation ou lui ayant peu ou prou survécu. C'est particulièrement regrettable pour les développements de S. Küchler (2002: 212, index, s.v. «Skin »), probablement les plus profonds et recevables jamais formulés à ce sujet, en vue de lier l'invention plastique malangan à des conceptions ou des perceptions très répandues de l'épiderme humain, au moins en Mélanésie. Sous cet aspect qu'il n'aborde pas directement, le catalogue résiste assez bien au dogmatisme occidental (« anti-mélanésien » pourrait-on dire aussi) par sa multiplicité de points de vue, quoiqu'il y sacrifie parfois par d'autres côtés, chaque chercheur étant inévitablement tenté de présenter son terrain d'étude comme le principal foyer de création ayant inspiré les autres, non sans effets " collatéraux ». Ce genre de réputation attaché de longue date aux îles Tabar a ainsi conduit nombre de marchands d'art primitif à orner d'une telle provenance des objets sans doute issus d'autres centres de style (notamment Jacques Kerchache, Îles Tabar, Paris, 1971, nom et référence curieusement absents de ce catalogue édité par le musée qu'il a « suscité »3).

3. Quoique la demande leur en ait été faite et réitérée, ni le musée ni l'éditeur n'ont consenti à faire le service de ce catalogue à notre société, ce qui est anormal, même si le $J S O$ peut s'honorer en cette occasion et une fois de plus d'informer ses lecteurs des « ouvrages non reçus ». 
L'autre difficulté majeure est celle de l'interprétation, rarement séparable de la précédente. Cet ouvrage en souligne effectivement l'importance en présentant, on l'a dit, deux éclairages distincts sur quelques thèmes ou types d'objets. Néanmoins, objectifs et méthodes nécessiteraient d'être plus précisément définis. Parmi les premiers, on voit mal qui pourrait contester cet idéal formulé par Gerd Koch (2003: 12), une compréhension des objets, de leur création et de leur emploi aussi fidèle que possible aux conceptions des autochtones qui les ont créés ou mis en œuvre - avec tout le « dépaysement » de la pensée que cela suppose de la part de chercheurs vivant et travaillant en Occident. De cette idée régulatrice découlent largement les méthodes d'enquête, d'analyse et de «lecture » en termes « modernes » inévitablement déformants et exigeant des corrections optiques qui les rendent moins inexacts ; leur mise au point - forcément empirique pour de multiples raisons impossibles à détailler ici - ne peut se passer d'une revue de toutes les «lectures» antérieures en vue de déterminer ce qu'elles contiennent de justesse ou de fausseté, pour en tirer le meilleur parti. De ce point de vue aussi, ce catalogue manque parfois de la profondeur historique voulue.

Deux exemples le montrent assez bien. Dans l'exposé qu'il consacre à ces « symboles de puissance et de pouvoir » que seraient les kapkap (56-57), Graeme Were assure, sans indication de source ni de contexte géographique ou historique (emploie-t-il un présent " ethnographique » ou d'actualité ? ambiguïté trop fréquente dans ce catalogue), qu'ils "ne sont montrés publiquement qu'à l'occasion des cérémonies mortuaires ", et seulement arborés par les chefs de clan, «d'habitude des hommes », dont ils signaleraient l'autorité un peu comme les barrettes d'officier aident à distinguer, sur les uniformes, un chef de bataillon d'un chef de peloton : « La taille du disque et le nombre de bandes concentriques figurées sur le morceau d'écaille de tortue symbolisent le nombre de clans dans lesquels son détenteur a été initié et au nom desquels il peut parler ». On regrette que l'interprétation astronomique avancée après d'autres par $\mathrm{S}$. Küchler (2002 : 53-54 et 118, « pattern said to represent the star wesburut ») ne soit même pas mentionnée. Mais outre le fait que des kapkap de taille plus réduite pouvaient être portés par les femmes, comme l'a rappelé Tibor Bodrogi (1961: $50-65$, p. 52, article non cité par le catalogue), et que maintes photos anciennes attestent leur port hors de tout contexte cérémoniel, les 123 objets néo-irlandais de ce type rassemblés et publiés par Gladys A. Reichard (1933 : II, pl. CV-CXX, $n^{\circ}$ 344-466, voir aussi I, 101, fig. 57 et texte, ouvrage non cité dans le catalogue) présentent une telle variété dans les motifs de ces « bandes concentriques» que le kapkap présenté en regard (ou à l'appui ?) de cette interprétation s'avère de dessin trop simple pour illustrer une question aussi complexe et sans doute toujours à élucider.

Pour sa part, M. Gunn se fait l'écho des explications qu'il a personnellement recueillies auprès d'une vieille autochtone à propos de sept panneaux peints représentant des figures féminines aux jambes fléchies et aux bras levés (avec des kapkap sur la poitrine peu compatibles avec l'interprétation de G. Were) ayant orné l'intérieur d'une maison d'initiation des jeunes filles, collectés par Walden et conservés au musée de Berlin (pp. 64-67). Il les résume ainsi : « Bien que décrits à l'origine comme des 'images liées à la mort appelées bongas', une femme d'un village voisin de Lakuramau les a identifiés plus récemment comme des panneaux représentant plusieurs stades de [la] maturité sexuelle d'une femme ». Selon une autre interprétation récente, développée par Marianne George (Assemblage, o. c. : 91-93; le catalogue ne cite ni le nom ni les travaux de cette spécialiste du pays Barok), cet ensemble « depicts the debutante-like display of young women, who are called dawan in Barok and Patpatar languages and davar in Mandak » dont les différences d'ornementation tiendraient au clan et à la moitié auxquelles elles appartenaient. On trouverait difficilement lectures plus opposées, et elles auraient certainement excité la verve de Victor Hugo : la première veut ordonner ces panneaux en une série diachronique où reconnaître la figuration " radiographique » (ou échographique) de quelques étapes du développement physiologique d'un quelconque individu féminin, comme des organisations d'éducation à la santé « universelle » en conçoivent pour l'édification des femmes du monde entier, la seconde $\mathrm{y}$ voit une représentation synchronique de jeunes femmes aux peintures corporelles variées, à fleur de peau, "superficielles » du point de vue occidental, mais probablement plus significatives d'une société particulière, soucieuse de magnifier ses spécificités et les relations qu'elle privilégiait entre femmes et hommes, corps et esprits qui la constituaient, par le truchement de ce «maquillage» dont Baudelaire pourrait avoir génialement saisi ce qu'il avait de « sauvage » en même temps que d'anti-naturel ou d'«artificiel ».

Au-delà des conceptions qu'on peut se faire en Occident de la « pensée sauvage » et de celles qui ont prétendu la penser, ou du choix (sans doute 
sous-tendu par les précédentes) entre ces lectures diachronique ou synchronique, universaliste ou particulariste, "radiographique » ou " épidermique » (pour suivre $\mathrm{S}$. Küchler), également applicables en apparence à d'autres séries d'objets néo-irlandais voués à être exposés conjointement (figures uli, sculptures des cases malangan, panneaux ornant les maisons des hommes, $c f$. pl. 54 pp. 170-171, masques, pierres sculptées, etc.), la méthode même recommanderait de soumettre plus systématiquement l'interprétation de ces arts à l'adage Lectio difficilior potior. Selon ce principe, que les philologues européens, au moins depuis Jean Le Clerc (Ars Critica, Amsterdam, 1689 : II, 293), appliquent à « l'établissement» des textes grecs, latins ou hébraïques formant la tradition occidentale " classique », et que leur ont emprunté nombre d'historiens des arts européens, entre diverses versions concurrentes d'un même énoncé ancien, la plus ardue à saisir, ou la plus éloignée de la première "solution" venue, serait aussi la plus vraisemblable sinon la plus proche de l'original, compte tenu de la tendance à la « banalisation », à l'aplatissement et aux contresens de toute sorte des copistes et exégètes postérieurs. La connaissance factuelle des arts néo-irlandais s'est considérablement enrichie ces dernières décennies grâce aux nouvelles technologies et au travail inlassable de nombreux chercheurs et conservateurs, M. Gunn le premier, dont cette exposition atteste et magnifie les efforts, jusqu'à cet appendice du catalogue présentant une liste inédite des principaux musées dépositaires de ces arts (pp. 283-291). Mais cet accroissement quantitatif sans précédent du savoir resterait de peu de prix s'il ne s'accompagnait d'un net progrès qualitatif dans l'intelligence de ces expressions artistiques.

De ce dernier point de vue, l'exposition, telle qu'elle fut présentée à Paris, était remarquablement accueillante et ouverte à toutes les « lectures ", faciles ou difficiles, immédiates ou différées. Les panneaux de présentation, sobres et peu nombreux, ne retenaient pas le regard au détriment des objets, comme il arrive trop souvent. Les cartels très concis apportaient souvent des nuances ou des précisions utiles (ainsi du kapkap déjà mentionné et des petits uli de faîtage ornant le sommet de huttes cérémonielles, dont la destination n'est mentionnée ni dans le texte ni dans les notices du catalogue, 172-174 et pl. 59-60). Les espaces d'exposition, habilement répartis de part et d'autre de la plus vaste salle consacrée aux immenses sculptures malangan, suggéraient discrètement les liens subtils unissant beaucoup de ces objets, tout en permettant d'en admirer les variations de style et d'expres- sion. Rompant avec l'absurde vogue des fonds noirs (encore sensible dans l'exposition des collections permanentes du musée, et même dans le catalogue) et du faux mystère des pénombres traversées d'avares filets de lumière, les maîtres d'œuvre avaient retenu une scénographie d'apparence assez neutre, mais convenant largement aux plus spectaculaires des objets exposés : cloisons rappelant par endroits les palissades d'enclos malangan, parois, sols et socles d'un ton sable chaud, légèrement ocré (pouvant évoquer l'ocre importé d'Anir décorant les sculptures malangan), éclairages généreux, circulation aisée sous une belle hauteur de plafond, ces choix n'étaient pas sans rapport avec certains paysages de Nouvelle-Irlande. Quant aux objets euxmêmes, souvent à couper le souffle, quoique bien connus pour la plupart, ils n'avaient jamais été réunis avec une telle profusion. Quel luxe de voir l'une à côté de l'autre les deux plus belles barques malangan au monde! Fait rarissime dans les expositions actuelles, on pouvait même quelquefois en faire le tour ou en examiner le détail sans l'obstacle d'une vitrine ou de ses reflets. Si l'on regrettait d'abord qu'ils dussent repartir, sans rien laisser d'autre à Paris qu'un souvenir émerveillé (pour des raisons « historiques », les collections du musée du quai Branly comptent peu d'objets comparables), on en venait à se remémorer ce qui caractérise une fête malangan, de brèves journées d'effervescence et de splendeur concluant des mois ou des années de préparation et de labeur, soit aussi le temps qu'il a fallu aux organisateurs de cette exposition pour réussir à honorer si dignement ces arts qui visent généralement à frapper les regards à vie, au moyen des œuvres ou des coups d'œil les plus éphémères.

\section{BIBLIOGRAPHIE}

Bodrogi Tibor, 1961. Kapkap in Melanesien, in Dietrich Drost und Wolfgang KöNIG (Hrsg), Beiträge zur Völkerforschung. Hand Damm zum 65. Geburtstag, Berlin, Akademie-Verlag, pp. 50-65.

DANKs Benjamin, 1933. In wild New Britain. The Story of Benjamin Danks, pioneer missionary, from his diary, ed. Wallace Dace, Sydney, Angus \& Robertson.

FOSTER Robert J., 1995. Social Reproduction and History in Melanesia. Mortuary ritual, gift exchange and custom in the Tanga Islands, Cambridge, Cambridge University Press.

GunN Michael, 1997. Arts rituels d'Océanie. NouvelleIrlande dans les collections du musée BarbierMueller, Milan, Skira. 
GunN Michael et Philippe Peltier (éds.), 2007. Nouvelle-Irlande. Arts du Pacifique Sud, avec des contributions de Vicky Barnecutt, Antje Denner, Brigitte Derlon, Sean Kingston, Susanne Küchler, Markus Schindlbeck et Graeme Were, Paris-Milan, musée du quai Branly - 5 Continents éditions, 304 p., 184 ill., cartes, bibliographie, index.

HeINTzE Dieter, 1969. Ikonographische Studien zur Malanggan-Kunst Neuirlands. Untersuchungen an ausgewählten Vogeldarstellungen, Tübingen, Universität Tübingen.

_, 2003. «Eine machtvolle Sache bei uns», $\mathrm{Zu}$ den Malagan des nördlichen Neuirland, in Wulf KöPFE und Bernd Schmelz (Hrsg.), Hamburg: Südsee. Expeditionen ins Paradies, Mitteil. aus dem Museum für Völkerkunde Hamburg, NF, Bd 33, pp. 155-194.

Helfrich Klaus, 1973. Malanggan I, Berlin, Museum für Völkerkunde.

JACQUES Norbert, 1922. Südsee. Eine Reisebuch, Drei Masken Verlag, Munich.

Koch Gerd, 2003. L'Océanie hier et aujourd'hui. Propos recueillis par Gilles Bounoure. Arts d'Afrique Noire-arts premiers, $\mathrm{n}^{\circ} 127$, pp. 8-15.

KräMER Augustin, 1925. Die Malanggane von Tombara, Munich, Georg Muller.

KRÄMER-BANNOw Elisabeth, 1916. Bei Kunstsinnigen Kannibalen der Südsee, Berlin, Dietrich Reimer.

KraUSE Fritz, 1907. Zur Ethnographie der Insel Nissan, Jahrb. des Städt. Museums für Völkerkunde zu Leipzig, Bd I, pp. 44-159.
Küchler Susanne, 2002. Malanggan : Art, Memory and Sacrifice, Oxford, Berg.

LinCOLN Louise (éd.), 1987. Assemblage of Spirits, Idea and Image in New Ireland, New York, Braziller.

MeINICKe Carl E., 1875. Die Inseln des Stillen Oceans, Leipzig, Frohberg.

Meyer Anthony J. P., 1995. Art océanien, Paris, Gründ.

Newton Douglas, Adrienne L. KAEPPLER et Christian Kaufmann, 1993. L'Art océanien, Paris, Mazenod.

Nicolas Alain et al., 2000. Art papou. Austronésiens et Papous de Nouvelle-Guinée, Marseille, Musées de Marseille.

PARKINSON Richard, 1907. Dreissig Jahre in der Südsee, Stuttgart, Strecker \& Schröder.

PowDERMAKER Hortense, 1933. Life in Lesu, London, William \& Norgate.

_, 1966. Stranger and Friend. The Way of an Anthropologist, New York, Norton.

ReICHARD Gladys A., 1933. Melanesian Design. A study of style in wood and tortoiseshell carving, New York, Columbia University Press.

StEPHAN Emil, 1907. Südseekunst, Berlin, Dietrich Reimer.

StePHAN Emil et Fritz GräBNER, 1907. NeuMecklenburg, Berlin, Dietrich Reimer. 